\title{
SA-VFI: the IEEE IUS challenge on Synthetic Aperture Vector Flow Imaging
}

\author{
Jensen, Jørgen Arendt; Liebgott, Herve; Cervenansky, Frederic; Villagómez Hoyos, Carlos Armando
}

Published in:

Proceedings of 2018 IEEE International Ultrasonics Symposium

Link to article, DOI:

10.1109/ULTSYM.2018.8580208

Publication date:

2018

Document Version

Peer reviewed version

Link back to DTU Orbit

Citation (APA):

Jensen, J. A., Liebgott, H., Cervenansky, F., \& Villagómez Hoyos, C. A. (2018). SA-VFI: the IEEE IUS challenge on Synthetic Aperture Vector Flow Imaging. In Proceedings of 2018 IEEE International Ultrasonics Symposium [8580208] IEEE. https://doi.org/10.1109/ULTSYM.2018.8580208

\section{General rights}

Copyright and moral rights for the publications made accessible in the public portal are retained by the authors and/or other copyright owners and it is a condition of accessing publications that users recognise and abide by the legal requirements associated with these rights.

- Users may download and print one copy of any publication from the public portal for the purpose of private study or research.

- You may not further distribute the material or use it for any profit-making activity or commercial gain

- You may freely distribute the URL identifying the publication in the public portal 


\title{
SA-VFI: the IEEE IUS challenge on Synthetic Aperture Vector Flow Imaging
}

\author{
Jørgen Arendt Jensen ${ }^{1}$, Hervé Liebgott ${ }^{2}$, Frederic Cervenansky ${ }^{2}$, Carlos Armando Villagomez Hoyos ${ }^{1}$ \\ ${ }^{1}$ Center for fast Ultrasound Imaging, Dept. Elec. Eng., Technical University of Denmark \\ 2 CREATIS, Univ Lyon, INSA-Lyon, Université Claude Bernard Lyon 1, UJM-Saint Etienne, \\ CNRS, Inserm, UMR 5220, U1206, Lyon, France
}

\begin{abstract}
High frame rate vector flow imaging represents the future way to implement velocity estimation modes on clinical systems. Several approaches to obtain such imaging modes are possible especially using plane or diverging waves. The SAVFI (Synthetic Aperture-Vector Flow Imaging) challenge is a competition organized during the IEEE International Ultrasonics Symposium 2018 in Kobe that aimed at bringing together all groups willing to work on synthetic aperture based high frame rate vector flow imaging This document describes the framework and objectives of the challenge as well as the data-sets and metrics used in the competition. The paper concludes with a discussion of the experiences from organizing this challenge.
\end{abstract}

Index Terms-synthetic aperture, flow, ultrafast, high frame rate, ultrasound

\section{INTRODUCTION}

Given the positive response to the IUS 2016 challenge on plane wave imaging (PICMUS) [1], a challenge on synthetic aperture (SA) 2-D vector flow imaging (VFI) has been organized. Synthetic aperture, as plane wave imaging, is a parallel system technique utilizing broader beams to insonify a larger region, e.g spherical waves. The advantages of employing such parallel systems for estimating blood flow are many, foremost, they break the tie between frame rate, region of interest (ROI), and precision of the estimates [2]. In the literature, there exist a variety of parallel systems techniques that are capable of estimating the velocity of the blood flow [3], [4]. Most often such estimators are evaluated on different data sets, and this makes direct comparison of performance difficult. The purpose of the challenge therefore has three main purposes. Firstly to provide both simulated and measured state-of-the-art SA data sets for VFI with a precise reference. The second goal is to make possible a direct and fair comparison between different estimators developed in different research groups who have otherwise almost no chance to perform such comparison. The third goal is to increase awareness of the challenges using such data sets and hopefully inspire new research on estimators and SA sequences whether it be with spherical [5] or plane waves [6]. The challenge therefor consist of estimating blood flow velocities from both simulated and measured ultrasound RF element SA data.

The contestants are given a number of pre-beamformed data sets generated either from Field II [7], [8] simulations or from experiments performed with the SARUS system [9]. For each data set a spatial grid is provided to the participant, where they are required to estimate the vector velocity fields and to provide the organizers with their results through a dedicated web platform (https://challenge.creatis.insa-lyon.fr/IEEE_IUS_2018). During the challenge, information is also shared with the community through a website ((https://www.creatis.insalyon.fr/Challenge/IEEE_IUS_2018/) and a report describing the data [10]. Both the platform and the website are intended to be maintained even after the IUS conference. The results are then automatically compared to a reference to calculate some evaluation metrics and perform a ranking among them.

This paper presents the imaging sequence, the different data sets made available, and finally the evaluations criteria. It is concluded by a discussion of our our observations on organizing such a challenge.

\section{IMAGING SEQUENCE}

A SA imaging scheme modelled after the method suggested in [2] was used, where a number of low resolution images are acquired repeatedly to enable correlation of the combined high resolution images. The particular sequence was suggested in [11] and is developed for vessels close to the linear array transducer, where the diverging spherical emissions are concentrated in the region directly beneath the probe.

\section{A. Probe and excitation}

A 128-element linear array probe was selected for the challenge. The transducer coordinate system is defined following the convention defined in Field II [7], [8], where the $z$ coordinate increases with depth, $x$ is along the elements, and a right-handed coordinate system is used. The array has a measured center frequency of $8 \mathrm{MHz}$ and a $60 \%$ bandwidth. The transducer parameters and variables are given in Table I. The excitation waveform used consists of a tapered $(50 \%$ Tukey window) 3 cycles sinusoidal waveform, and the impulse response of the probe was supplied to the contestants.

\section{B. Duplex SA sequence}

The provided data are acquired with a linear array probe using a duplex sequence (both B-mode and flow). The sequence is repeated to generate continuous data, so that the data can 
TABLE I

PARAMETERS AND VARIABLES FOR THE TRANSDUCER USED.

\begin{tabular}{|l|c|c|l|}
\hline Parameter & Value & Unit & Matlab variable \\
\hline \hline Center freq. & 8 & $\mathrm{MHz}$ & xdc.f0 \\
\hline No. elements & 128 & elem. & xdc.n_elements \\
\hline Pitch & 0.3 & $\mathrm{~mm}$ & xdc.pitch \\
\hline Element height & 4 & $\mathrm{~mm}$ & xdc.height \\
\hline Elevation focus & 20 & $\mathrm{~mm}$ & xdc.elevation_focus \\
\hline Element pos. & {$[\mathrm{x} \mathrm{y} \mathrm{z}]$} & $\mathrm{m}$ & $\begin{array}{l}\text { xdc.element_positions } \\
(128 \mathrm{x} \text { 3) }\end{array}$ \\
\hline Impulse resp. & & $P a /\left(\mathrm{Vs}^{2}\right)$ & para.xdc.impulse_response \\
\hline
\end{tabular}

be beamformed throughout the whole imaging region, and are continuously available. The imaging sequence is as follows:

\begin{tabular}{l|c|c|c|c|c|c} 
Emission: & 1 & 2 & 3 & 4 & 5 & 6 \\
\hline Type: & $V_{1}$ & $V_{2}$ & $V_{3}$ & $V_{4}$ & $V_{5}$ & $B_{1}$ \\
\hline Emission: & 7 & $8-11$ & 12 & 13 & $14-767$ & 768 \\
\hline Type: & $V_{1}$ & $\ldots V_{5}$ & $B_{2}$ & $V_{1}$ & $\ldots V_{5}$ & $B_{128}$ \\
\hline
\end{tabular}

where $V_{1}$ is a velocity emission for virtual source 1 and $V_{5}$ is the emission equal to the number of velocity emissions. $B_{2}$ is a B-mode emissions for virtual source 2 . The inter-spaced Bmode sequence is 128 emissions long and starts again with $B_{1}$, when the last B-mode emission has been made, thus, having a frame size of 768 emissions. The sequence is the same as has been used in [11]. A summary of the transmit sequence parameters are listed in Table II.

TABLE II

ACQUISITION/SIMULATION PARAMETERS

\begin{tabular}{llc}
\hline \multicolumn{3}{c}{ Transmit Parameters } \\
\hline Parameter & B-mode & Flow \\
\hline Number of emitting elements & 16 & 64 \\
Tx Apodization window & \multicolumn{1}{c}{ Hanning } \\
F-number & -1 & -3.5 \\
Number of distinct beams & 128 & 5 \\
Excitation signal & 3 sinusoids with a \\
& $50 \%$ Tukey window \\
Pulse repetition frequency (PRF) & $5 \mathrm{kHz}$ \\
\hline
\end{tabular}

The placement of the various virtual emission centers are shown in Fig. 1. Note that some of the emissions for the Bmode image are only shown as a dot, and only the first 5 velocity emissions are shown. The following ones are placed at the same locations. The insonified area of virtual source \# 51, used for B-mode imaging, is shown in Fig. 2 (left). The placement of the transducer elements are shown in blue and the active elements for the emission are colored red. The two lines delineates the acceptance angle for the virtual source, so that the insonified area is present within them. Similarly the virtual source \#1 for blood flow velocity estimation is shown in Fig. 2 (right).

\section{SUPPLIED DATA}

The supplied data sets were shared through the SA-VFI challenge website. The participants were provided with the different data sets listed in Table III, which included in particular both simulations and experiments from a 90 degrees

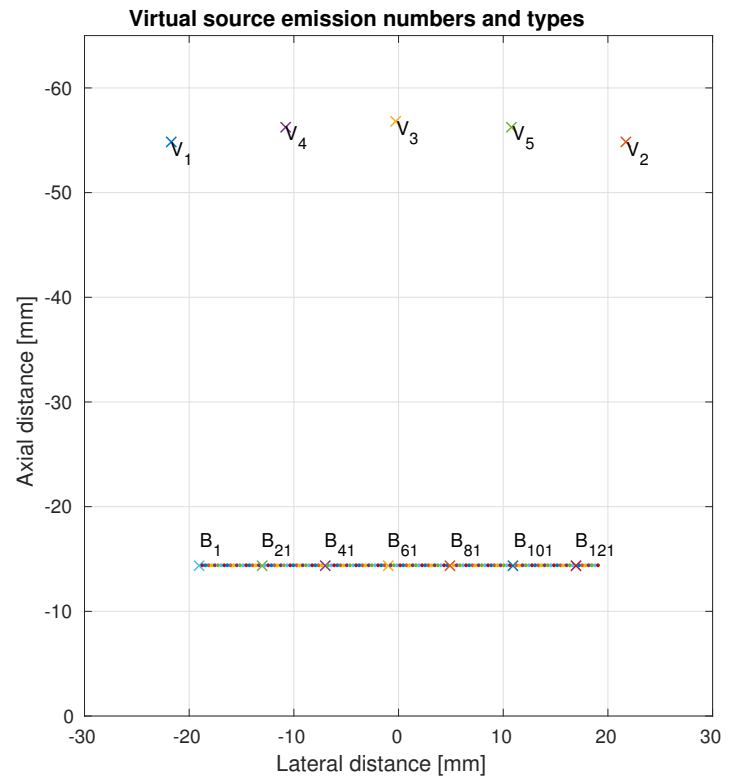

Fig. 1. Placement of virtual sources in the imaging sequence
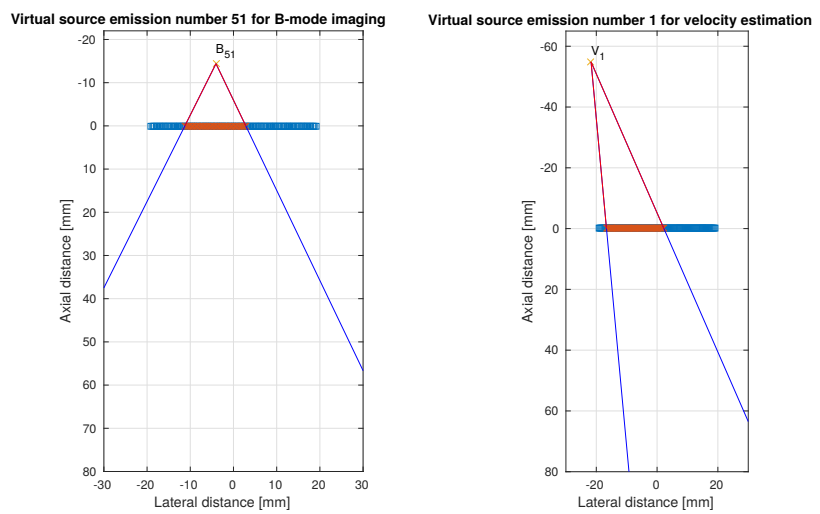

Fig. 2. Placement of virtual sources in the imaging sequence

and 105 degrees straight vessel as well as a simulated spinning disc. All data sets were available together with the corresponding ground truth values during the training period including one example data set obtained from a CFD simulation of a carotid bifurcation without reference. For testing a final CFD simulation was provided to the participants with the resulting velocity values blinded to the contestants.

\section{Evaluation in THE COMPETITION}

The performance was evaluated using global metrics. Bias and standard deviations were calculated for both the angle and the magnitude of the vector field as:

$$
\begin{gathered}
\sigma=\sqrt{\frac{\sum_{i=1}^{N}\left(\hat{m}(i)-m_{\text {true }}(i)\right)^{2}}{N^{2} m_{\text {peak }}}} \\
m_{\text {bias }}=\frac{\sum_{i=1}^{N}\left(\hat{m}(i)-m_{\text {true }}(i)\right)}{N m_{\text {peak }}} .
\end{gathered}
$$


TABLE III

PROVIDED DATA SETS

\begin{tabular}{|c|c|}
\hline Content & Description \\
\hline $\begin{array}{l}\text { Wire Phan- } \\
\text { tom }\end{array}$ & $\begin{array}{l}\text { A wire phantom containing } 5 \text { wires at depths of } 31,56 \text {, } \\
81,106 \text {, and } 131 \mathrm{~mm} \text { are both simulated in Field II, } \\
\text { and measured with using the SA sequence. The phantoms } \\
\text { are meant to be used as reference to verify beamforming } \\
\text { algorithms }\end{array}$ \\
\hline $\begin{array}{l}\text { Straight } \\
\text { vessel } \\
\text { @ } 105^{\circ}\end{array}$ & $\begin{array}{l}\text { Parabolic profile straight vessel phantoms with a beam- } \\
\text { to-flow angle of } 105^{\circ} \text { are both simulated and measured. } \\
\text { The simulated data set is obtained using Field II, where } \\
\text { stationary echoes are included from the vessel boundary } \\
\text { and are } 40 \mathrm{~dB} \text { larger than the blood scattering. The signal- } \\
\text { to-noise ratio in the vessel is } 15 \mathrm{~dB} \text {. The measured data set } \\
\text { is obtained from a flow rig circulating blood-mimicking } \\
\text { fluid. The length of the entrance tube is long enough } \\
\text { to ensure fully-developed laminar flow with a parabolic } \\
\text { profile. }\end{array}$ \\
\hline $\begin{array}{l}\text { Straight } \\
\text { vessel } \\
@ 90^{\circ}\end{array}$ & $\begin{array}{l}\text { A set of simulated and measured data for a straight vessel } \\
\text { phantom with a beam-to-flow angle of } 90^{\circ} \text {. The same } \\
\text { characteristics as Straight vessel @ } 105^{\circ} \text {. }\end{array}$ \\
\hline $\begin{array}{l}\text { Spinning } \\
\text { disc }\end{array}$ & $\begin{array}{l}\text { Data set simulated using Field II. The phantom rotates } \\
\text { clock-wise, having a diameter of } 1.5 \mathrm{~cm} \text {, and a maximum } \\
\text { velocity } v_{\max }=0.25 \mathrm{~m} / \mathrm{s} \text { at the outer edge. The center } \\
\text { is placed at a depth of } 2.5 \mathrm{~cm} \text { below the center line of the } \\
\text { array. The elevation extent of the spinning disk is } 5 \mathrm{~mm} \text {. } \\
\text { White noise is added to obtain a SNR of } 10 \text { in dB. }\end{array}$ \\
\hline CFD model & $\begin{array}{l}\text { Simulated pulsatile flow in a carotid bifurcation based on } \\
\text { CFD flow model developed by Swillens et al [12]. No } \\
\text { noise is added. The phantom is meant as an example for } \\
\text { the } 2 \text { nd stage of the challenge. }\end{array}$ \\
\hline
\end{tabular}

Here $m(i)$ is the estimated metric (angle or magnitude of the velocity) by the participant at a given point, $m_{\text {true }}(i)$ is the ground truth at the same point, and $m_{\text {peak }}$ is the maximum value attainable by the metric. The estimates are ordered in a vector that can be converted to a spatial and temporal position. For the angle circular statistics are used to avoid aliasing around $360^{\circ}$ angle.

The participants receive for each metric a number of points that depends on their position among all the participants results. The participant having the best result for a given metric receives 100 points and the one having the worst result receives 0 points. For each metric, a challenger obtaining a value in between receives a number of points calculated as follows:

$$
N_{\text {points }}=100 \cdot \frac{\text { Max }_{\text {metric }}-\text { Challenger }_{\text {metric }}}{\text { Max }_{\text {metric }}-\text { min }_{\text {metric }}}
$$

where Max $x_{m e t r i c}$ and min $_{\text {metric }}$ are the maximum and minimum values obtained among all participants for the current metric and Challenger ${ }_{m e t r i c}$ is the value obtained by the challenger considered in the current metric. This calculation is performed for all four metrics (bias and standard deviations for both angle and magnitude).

Finally all points obtained for all metrics and all data-sets are summed up, and the participant obtaining the maximum number of points is designated as the winner. Note that for the training all data-sets except the CFD were used, whereas for the final testing only the blinded CFD data-set was used. Contestants were asked to use the same code for both challenge stages.

\section{EXAMPLES}

A number of examples for beamforming and simple velocity estimation was given to the contestants for helping to start their contribution. This was described in the report for the competition along with the data sets and the evaluation criteria [10].

\section{A. Beamforming example}

The beamformed B-mode image for a straight vessel with a beam-to-flow angle of $90^{\circ}$ for both simulated and measured data sets are shown in the top of Fig. 3. In the same manner the B-mode for the spinning disk and carotid bifurcation model are shown in the bottom of Fig. 3. Since the performance of a beamformer is better shown as point spread functions of wires, a wire phantom is also provided as a reference.
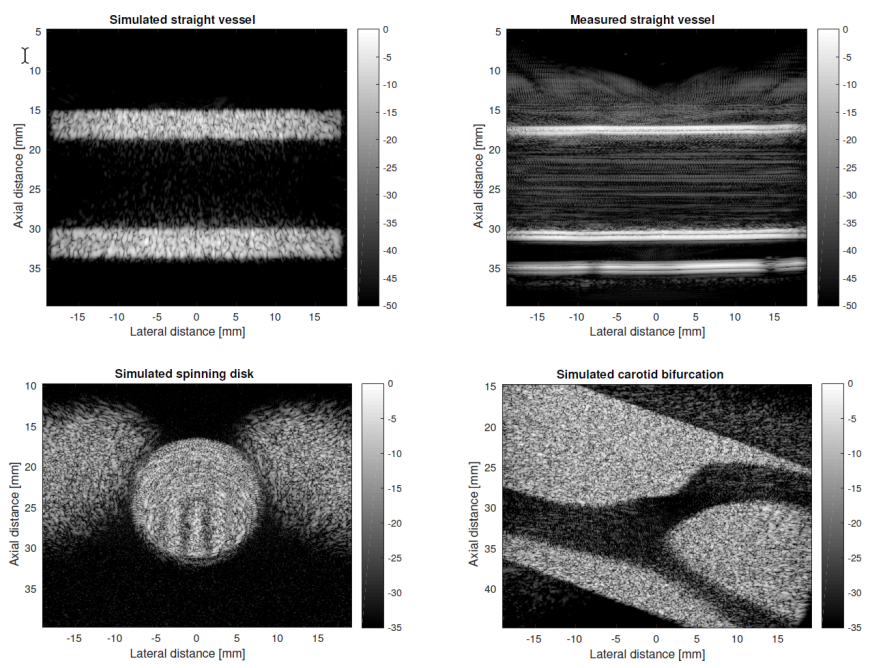

Fig. 3. Beamformed images of the data simulated and experimental straight vessel $105^{\circ}$ data-sets (top), and for the spinning disc (lower left) and the CFD trainingn set (lower right)

\section{B. Straight vessel}

An example plot for the $105^{\circ}$ vessel data set is shown in Fig. 4. The plot provides a graphical results for the mean, standard deviation, and true value for the evaluated magnitude and angle, while the numerical results used by the platform to score the participants are displayed in the screen.

\section{C. $C F D$}

An example evaluation plot for reference CFD data-set is shown in Fig. 5. This data-set was not used for evaluation in the challenge, however, results from another CFD blinded data-set was provided in the second stage and has been evaluated using the same metrics. The results are evaluated against the ground truth provided by the velocity field from the CFD model. 


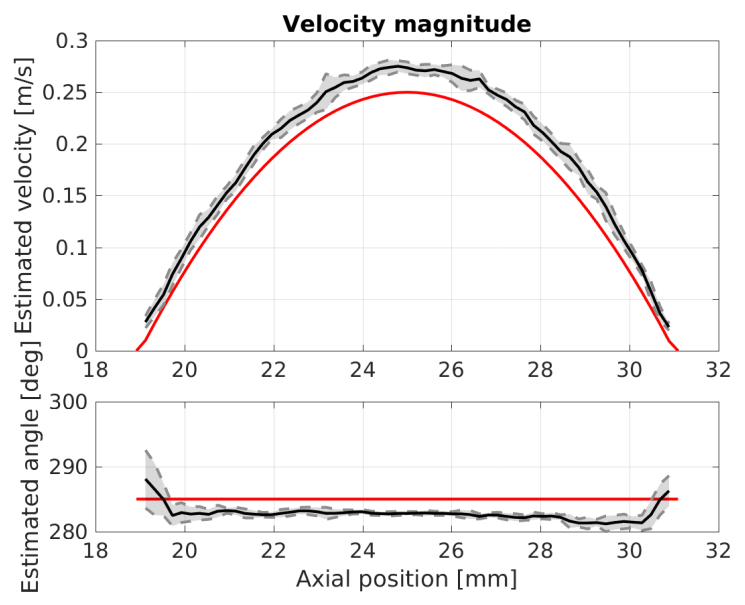

Fig. 4. Profile plots for the provided example result for the straight vessel phantom at 105 degrees. The true profiles are shown as the red line, while the mean and standard deviation are shown in black and grey, respectively. The top plot represents the magnitude and the bottom one represents the angle.
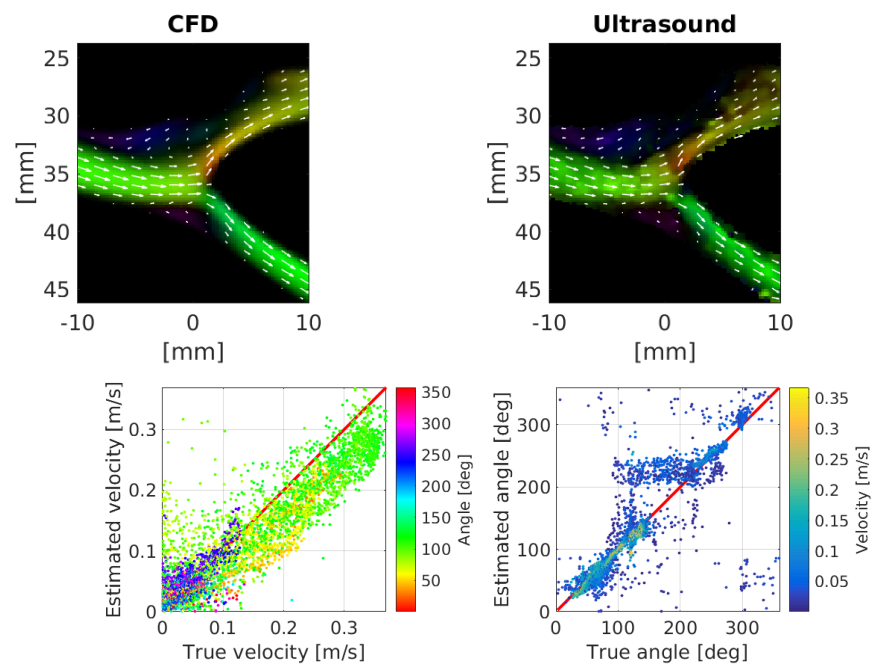

Fig. 5. Plots generated using the evaluation code on CFD example results. [Top] Vector flow imaging (VFI) frames from the reference CFD model (left), and the estimated velocity fields from ultrasound (right) during late systole. [Bottom] Scatter plots comparing the reference CFD values to the ultrasound estimates. (Left) The scatter plot of the estimated velocities color coded with the estimated angle. (Right)

\section{Final CFD DATA SET}

The final data set for evaluating the competition is based on the Swillens et al [12] simulation model for a carotid bifurcation with a simulation of the signals using Field II. The pulse repetition frequency $f_{p r f}$ was increased to $15 \mathrm{kHz}$ to make it possible to estimate the higher velocities in the bifurcation during the systolic phase. All other parameters are the same as for the other data sets. The geometry of the CFD model is shown in Fig. 6. The vessel is colored red and the box containing scatterers is green. The rectangular scan region is blue with an indication of the transducer placement in black. The sequence has only been simulated for the first 3 emissions sequence covering part of the cardiac cycle to not make the

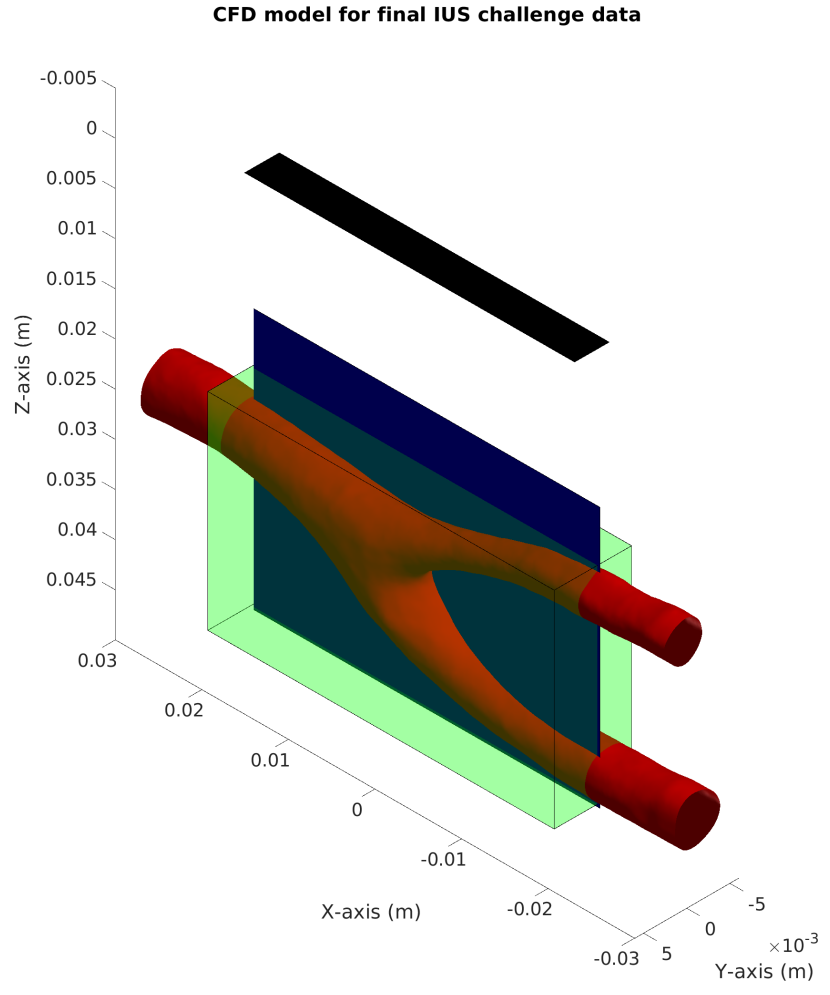

Fig. 6. Visualization of the final CFD model. The red model is the vessel, green is the box containing the scatterers, the black structure is the transducer, and blue is the scan plane.

data set too large.

\section{A. Final evaluation grid}

The velocity estimates for this CFD data set is evaluated on a rectangular grid with the following coordinates in Matlab:

$$
\begin{aligned}
& z=(18: 0.5: 40)^{\prime} / 1000 ; \\
& x=(-8: 0.5: 8) / 1000 ;
\end{aligned}
$$

where $z$ is in the depth direction and $x$ is lateral position. The grid, thus, starts from $18 \mathrm{~mm}$ and the estimates are found for every $0.5 \mathrm{~mm}$ down to $40 \mathrm{~mm}$. This is done for lateral positions from $-8 \mathrm{~mm}$ to $8 \mathrm{~mm}$ in a step of $0.5 \mathrm{~mm}$. Velocities outside the vessel are discarded in the evaluation.

The evaluation is performed at time 0.050 seconds from the start of the sequence where the first emission corresponds to time 0 . Data acquired both prior and past this time point can be used for finding the velocity estimate submitted to the competition.

\section{Discussion AND CONCLUSION}

Setting up a competition like PICMUS or SA-VFI is a real challenge. First of all, the topic should be broad enough to bring together enough potential competitors. This was definitely the case with high frame rate vector flow, which is one of the main research topics in the ultrasound community. If the synthetic aperture sequence could appear less straightforward as plane wave sequences, this was counterbalanced in SAVFI by the fact that some example codes were provided to the 
participants. The second difficulty is to provide the participants with data sets that are as realistic as possible compared to the in-vivo problem that we are willing to tackle. This is also a complicated task, since ground truth must be known in order to evaluate the participants scores, which is typically impossible for in-vivo data sets. Current state-of-the-art estimators are more precise with a higher temporal resolution than current "gold standard" MR measurements, and this precludes the use of in-vivo data as no adequately precise reference can be found. This leads to the use of simulations and in-vitro data with precisely known references, but which might sometimes be easier to process as tissue motion often is neglected.

Finally the rules used to determine the winner must by nature include mixing of metrics measuring different aspects. How these metrics are combined results in a compromise chosen by the organizers which might often be questioned. As a researcher in the field, what is probably more important is to describe and analyze all metrics separately instead of just finding out one winner out of a number of participants.

At the end challenges are a useful tool to structure the community and make specialists of a given domain exchange and compare their methods using the same data-sets and metrics. They represent a huge amount of work to organize, but make it possible for researchers who do not know each other to compare their techniques. It also provides a data set which in the future hopefully can be used as a reference for comparison.

\section{ACKNOWLEDGMENT}

The authors would like to thank Verasonics for their financial support. Hervé Liebgott is working within the framework of the LABEX PRIMES (ANR-11-LABX-0063) of Université de Lyon, within the program Investissements d'Avenir (ANR11-IDEX-0007) operated by the French National Research Agency (ANR). This work was financially supported by the Danish National Advanced Technology Foundation (82-20124), Innovation Fund Denmark (7050-00004B) and by BK Medical, Herlev, Denmark.

\section{REFERENCES}

[1] H. Liebgott, A. Rodriguez-Molares, F. Cervenansky, J.A. Jensen, and O. Bernard. Plane-wave imaging challenge in medical ultrasound. In Proc. IEEE Ultrason. Symp., pages 1-4, 2016.

[2] S. I. Nikolov and J. A. Jensen. In-vivo synthetic aperture flow imaging in medical ultrasound. IEEE Trans. Ultrason., Ferroelec., Freq. Contr., 50(7):848-856, 2003.

[3] J. A. Jensen, S. I. Nikolov, A. Yu, and D. Garcia. Ultrasound vector flow imaging I: Sequential systems. IEEE Trans. Ultrason., Ferroelec., Freq. Contr., 63(11):1704-1721, 2016.

[4] J. A. Jensen, S. I. Nikolov, A. Yu, and D. Garcia. Ultrasound vector flow imaging II: Parallel systems. IEEE Trans. Ultrason., Ferroelec., Freq. Contr., 63(11):1722-1732, 2016.

[5] J. A. Jensen, S. Nikolov, K. L. Gammelmark, and M. H. Pedersen. Synthetic aperture ultrasound imaging. Ultrasonics, 44:e5-e15, 2006.

[6] M. Tanter and M. Fink. Ultrafast imaging in biomedical ultrasound IEEE Trans. Ultrason., Ferroelec., Freq. Contr., 61(1):102-119, January 2014.

[7] J. A. Jensen and N. B. Svendsen. Calculation of pressure fields from arbitrarily shaped, apodized, and excited ultrasound transducers. IEEE Trans. Ultrason., Ferroelec., Freq. Contr., 39:262-267, 1992.
[8] J. A. Jensen. Field: A program for simulating ultrasound systems. Med. Biol. Eng. Comp., 10th Nordic-Baltic Conference on Biomedical Imaging, Vol. 4, Supplement 1, Part 1:351-353, 1996.

[9] J. A. Jensen, H. Holten-Lund, R. T. Nilsson, M. Hansen, U. D. Larsen, R. P. Domsten, B. G. Tomov, M. B. Stuart, S. I. Nikolov, M. J. Pihl, Y. Du, J. H. Rasmussen, and M. F. Rasmussen. Sarus: A synthetic aperture real-time ultrasound system. IEEE Trans. Ultrason., Ferroelec., Freq. Contr., 60(9):1838-1852, September 2013.

[10] J. A. Jensen and C. A. Villagomez-Hoyos. 2018 IEEE IUS SA-VFI Challenge: Version 2.0, August 27, 2018. Technical report, Dept. of Elec. Eng, Technical University of Denmark, 2018.

[11] C. A. Villagomez-Hoyos, M. B. Stuart, K. L. Hansen, M. B. Nielsen, and J. A. Jensen. Accurate angle estimator for high frame rate 2-D vector flow imaging. IEEE Trans. Ultrason., Ferroelec., Freq. Contr, 63(6):842-853, 2016.

[12] A. Swillens, L. Løvstakken, J. Kips, H. Torp, and P. Segers. Ultrasound simulation of complex flow velocity fields based on computational fluid dynamics. IEEE Trans. Ultrason., Ferroelec., Freq. Contr., 56(3):546556, 2009. 